\title{
A New Approach to Fault Diagnosis of Power Systems Using Fuzzy Reasoning Spiking Neural P Systems
}

\author{
Guojiang Xiong, Dongyuan Shi, Lin Zhu, and Xianzhong Duan \\ State Key Laboratory of Advanced Electromagnetic Engineering and Technology, Huazhong University of Science and Technology, \\ Wuhan, Hubei 430074, China \\ Correspondence should be addressed to Dongyuan Shi; dongyuanshi@hust.edu.cn
}

Received 24 January 2013; Accepted 9 May 2013

Academic Editor: Sabrina Senatore

Copyright (C) 2013 Guojiang Xiong et al. This is an open access article distributed under the Creative Commons Attribution License, which permits unrestricted use, distribution, and reproduction in any medium, provided the original work is properly cited.

Fault diagnosis of power systems is an important task in power system operation. In this paper, fuzzy reasoning spiking neural P systems (FRSN P systems) are implemented for fault diagnosis of power systems for the first time. As a graphical modeling tool, FRSN P systems are able to represent fuzzy knowledge and perform fuzzy reasoning well. When the cause-effect relationship between candidate faulted section and protective devices is represented by the FRSN P systems, the diagnostic conclusion can be drawn by means of a simple parallel matrix based reasoning algorithm. Three different power systems are used to demonstrate the feasibility and effectiveness of the proposed fault diagnosis approach. The simulations show that the developed FRSN P systems based diagnostic model has notable characteristics of easiness in implementation, rapidity in parallel reasoning, and capability in handling uncertainties. In addition, it is independent of the scale of power system and can be used as a reliable tool for fault diagnosis of power systems.

\section{Introduction}

Along with the unceasing development of electric power industry, the scale of power system is expanding constantly, and the complexity of network structure is increasing continuously. Under this situation, the occurrence of a fault will have great influence upon the normal operation of a power system. Unfortunately, fault is inevitable during the operation of power system. When fault events occur, it is imperative to isolate the faulted section or sections from the healthy part of the power system and limit the impact of power supply interruption to a minimum as soon as possible. To achieve this goal, the fast and accurate identification of the faulted section or sections is of great significance and is the main issue of the fault diagnosis of power systems.

The fault diagnosis of power systems is a process that identifies a faulted section or sections using a set of operational information of protective relays (PRs) and circuit breakers (CBs) available from the supervisory control and data acquisition (SCADA) systems. The process is difficult and complicated. The complexity will increase significantly when involving failed and/or malfunctioned PRs and/or CBs, multiple faults, or even a concurrence of them. It is because of these contingencies that the fault information has the characteristics of incompleteness and uncertainty. To solve this serious issue, various approaches have been proposed, including expert systems (ES) [1-3], artificial neural networks (ANNs) [4-9], Bayesian networks (BNs) [10-12], Petri nets (PNs) [13-17], Cause-Effect nets (CE-Nets) [18, 19], and analytic optimization model (AOM) [20-25]. ES use a rulebased knowledge base and a reasoning engine to identify the faulted sections. Although they are a relatively early and widely used approach for fault diagnosis, some inherent drawbacks, such as maintenance difficulties, slow response time, and weak fault tolerance, exist in them. Compared to ES, ANNs have a better fault tolerance and faster response time, but they behave following an opaque black box device model which functioning is unknown by the dispatchers. In addition, extracting numerous representative training samples and determining ANNs' structure parameters are tedious and tough jobs. In contrast to ANNs, BNs can explicitly represent the causalities between faulted section and the operations of protective devices. However, extracting training samples is still required to determine the structure parameters. Although PNs and CE-Nets do not need the training 
procedure, they have their own disadvantages. For example, PNs only have two types of nodes (place and transition) making PNs not able to represent the causalities perfectly and directly. Forward reasoning strategy cannot enable CE-Nets to visually represent all possible combinations of main, primary backup, and secondary backup protection operations for reasoning a fault. Additionally, CE-Nets' fault tolerance remains to be improved. With regard to the AOM, other than the graphic based approaches, it constructs an analytic model according to the operating principles of protection system to reflect the difference between the expected and the practical states of protective devices and then obtains the diagnostic results via minimizing the difference with the help of different optimization methods. However, the construction of satisfactory analytic models and the design of competent optimization methods are two major challenges to AOM.

Spiking neural P systems (SN P systems), which were firstly introduced in [26] in the framework of membrane computing [27], are a new class of distributed and parallel computing devices. They are inspired by the neurophysiological behavior of neurons sending electrical impulses (spikes) along axons to other neurons. An SN P system can be viewed as a set of neurons placed in the nodes of a directed graph whose arcs represent the synaptic connections among the neurons. The flow of information stream is realized by pulse potentials encoded by spikes (contained in neurons and denoted in what follows by the symbol $a$ ). This stream can be sent from presynaptic to postsynaptic neurons according to specific rules. Since the introduction, various SN P systems have been proposed, one of which is fuzzy SN P systems (FSN $\mathrm{P}$ systems) [28, 29]. The FSN P systems were proposed to represent fuzzy knowledge and model dynamic fuzzy reasoning process. However, the models in $[28,29]$ are too simple to perform fuzzy reasoning in parallel through matrix based operation. For conquering this shortage, the authors of [30] proposed a more systematic and comprehensive FSN P system, that is, fuzzy reasoning SN P systems (FRSN P systems), and developed a parallel fuzzy reasoning algorithm through matrix based operation according to neuron's dynamic firing mechanism. The FRSN P systems can visually represent fuzzy production rules of a fuzzy knowledge base and effectively model the corresponding dynamic fuzzy reasoning behavior.

Based on the aforementioned prominent features, this paper attempts to apply the FRSN P systems to the fault diagnosis of power systems. The contribution of this paper includes three folders.

(1) To our knowledge, there have been no reports about implementing the FRSN P systems for solving fault diagnosis of power systems. Thus, the first and most important contribution is to implement the FRSN P systems for fault diagnosis of power systems for the first time and study how they solve this important problem.

(2) A diagnostic framework based on the FRSN P systems is developed. This framework is able to make the
FRSN P systems based diagnostic model have good flexibility and extensibility.

(3) The operation statistics coupled with experience are used to tackle the uncertainties of PRs and CBs. They are able to realistically reflect the uncertainties among the PRs, CBs, and power system sections and thus make the developed FRSN P systems based diagnostic model more practical.

The structure of the paper is organized as follows. Section 2 describes the fault diagnosis of power systems problem. Section 3 recalls the definition of FRSN P systems and their matrix based reasoning algorithm. The proposed FRSN P systems based diagnostic framework and diagnostic models are elaborated, and some key issues in implementation of FRSN P systems for fault diagnosis of power systems are discussed in Section 4. In Section 5, the FRSN P systems based diagnosis approach is verified through three different applications, and Section 6 concludes this paper.

\section{Problem Description}

Because of nature disturbing or man-made influence, power systems are threatened by the occurrence of faults during the operation. When a fault occurs in a power system, the well-designed protection system quickly detects the fault and activates its PRs to trip the corresponding CBs to clear the fault. In the clearance process, there are uncertainties regarding the protective devices, such as failed, malfunctioned operation of PRs and/or CBs. To guarantee that the faulted section is completely isolated within a given amount of setting time, multiprotection configuration is adopted. In general, protection system consists of main protection and backup protection. When a fault occurs on a section, the section's main protection is firstly activated to isolate the fault. If the main protection fails, the backup protection must operate to eliminate the fault. In order to illustrate the concept of fault diagnosis, a simple power system [31] shown in Figure 1 is presented as a study case. Suppose that a fault, shown in Figure 1(a), occurs on line L1 and causes the actions of main PRs L1Am and L1Cm to trip the circuit breakers CB1 and CB2, respectively. If $\mathrm{CB} 1$ succeeds in opening while $\mathrm{CB} 2$ fails, the local backup of $\mathrm{L1Cm}$, that is, the primary backup PR L1Cp, operates to trip CB2 to isolate the fault. And if CB2 is not tripped again, the remote backups of $\mathrm{L} 1 \mathrm{Cm}$ and $\mathrm{L1Cp}$, that is, the secondary backup PRs L2Bs and L3Ds, operate to trip the circuit breakers CB3 and CB6, respectively, to successfully clear the fault. For the bus section, the fault clearance process is somewhat simpler. As shown in Figure 1(b), if a fault occurs on bus $\mathrm{C}$, its own main PR Cm will operate to send a signal to trip the circuit breakers CB2, CB4, and CB5. If some problems result in the failure of the main protection, the secondary backup protection will operate. For example, if $\mathrm{Cm}$ operates while CB5 fails to open, the secondary backup PR L3Ds will operate to successfully trip the circuit breaker CB6, and the fault is cleared. These fault information, that is, the alarms of the operated protective devices, will then be transmitted to the SCADA system. From the SCADA system, these available alarms are exploited to identify the faulted section or sections. 


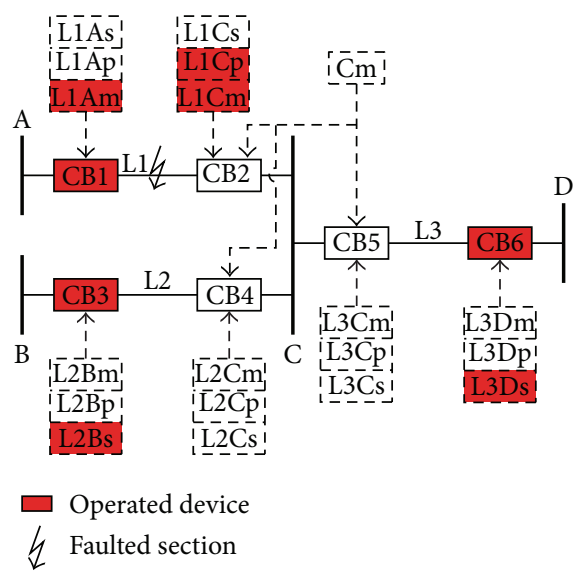

(a)

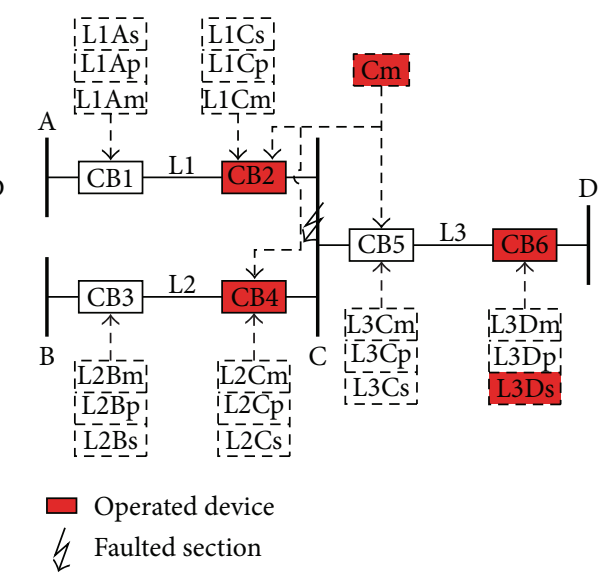

(b)

FIGURE 1: A simple power system. m: main protective relay; p: primary backup protective relay; s: secondary backup protective relay. (a) Line L1 fault; (b) bus C fault.

\section{Fuzzy Reasoning Spiking Neural P Systems}

\subsection{Definition}

Definition 1. A FRSN P system of degree $m \geqslant 1[28,30]$ can be defined as a 6 -tuple

$$
\Pi=\left(A, N_{p}, N_{r}, \text { syn, } I, O\right),
$$

where

(1) $A=\{a\}$ is the singleton alphabet (the object $a$ is called spike);

(2) $N_{p}=\left\{\sigma_{p 1}, \sigma_{p 2}, \ldots, \sigma_{p m}\right\}$ is called proposition neuron set, where $\sigma_{p i}$ is its $i$ th proposition neuron associated with a fuzzy proposition in a fuzzy knowledge base, $1 \leq i \leq m$. Each proposition neuron $\sigma_{p i}$ has the form $\sigma_{p i}=\left(\alpha_{p i}, \theta_{p i}, \lambda_{p i}, \beta_{p i}, b_{p i}, \mathrm{r}_{p i}\right)$, where

(a) $\alpha_{p i} \in[0,1]$ and it is called the (potential) value of spike contained in neuron $\sigma_{p i}$. It is used to express fuzzy truth value of $i$ th proposition neuron. The initial truth value is denoted by $\alpha_{p i}^{0}$;

(b) $\theta_{p i}$ is an integer and represents the number of spikes received by $i$ th proposition neuron. The initial number of spikes received is denoted by $\theta_{p i}^{0}$

(c) $\lambda_{p i}$ is an integer and represents the number of spikes required by firing $i$ th proposition neuron;

(d) $\beta_{p i} \in[0,1]$ and represents fuzzy truth value exported by $i$ th proposition neuron after firing;

(e) $b_{p i} \in\{0,1\}$ and represents the number of spikes exported by $i$ th proposition neuron after firing;

(f) $\mathrm{r}_{p i}$ is a firing/spiking rule contained in $i$ th proposition neuron, of the form $E / a^{x} \rightarrow a^{y}$, where $x, y \in[0,1]$;

(3) $N_{r}=\left\{\sigma_{r 1}, \sigma_{r 2}, \ldots, \sigma_{r n}\right\}$ is called rule neuron set, where $\sigma_{r i}$ is its $i$ th rule neuron associated with a fuzzy production rule in a fuzzy knowledge base, $1 \leq i \leq n$. $N_{r}$ contains two types of rule neurons, "AND" type neuron and "OR" type neuron. Each rule neuron $\sigma_{r i}$ has the form $\sigma_{r i}=\left(h_{r i}, g_{r i}, \alpha_{r i}, \theta_{r i}, \lambda_{r i}, \beta_{r i}, b_{r i}, \gamma_{r i}, \mathrm{r}_{r i}\right)$, where

(a) $h_{r i}, g_{r i} \in\{0,1\}$. If the $i$ th rule neuron is an "AND" type neuron, $h_{r i}=1, g_{r i}=0$; otherwise, $h_{r i}=0, g_{r i}=1$;

(b) $\alpha_{r i} \in[0.1]$ and it is called the (potential) value of spike contained in neuron $\sigma_{r i}$. The initial truth value is denoted by $\alpha_{r i}^{0}$;

(c) $\theta_{r i}$ is an integer and represents the number of spikes received by $i$ th rule neuron. The initial number of spikes received is denoted by $\theta_{r i}^{0}$;

(d) $\lambda_{r i}$ is an integer and represents the number of spikes required by firing $i$ th rule neuron;

(e) $\beta_{r i} \in[0,1]$ and represents fuzzy truth value exported by $i$ th rule neuron after firing;

(f) $b_{r i} \in\{0,1\}$ and represents the number of spikes exported by $i$ th rule neuron after firing;

(g) $\gamma_{r i} \in[0,1]$ and represents confidence factor of $i$ th production rule associated with rule neuron $\sigma_{r i}$

(h) $\mathrm{r}_{r i}$ is a firing/spiking rule contained in ith rule neuron, of the form $E / a^{x} \rightarrow a^{y}$, where $x, y \in$ $[0,1]$

(4) syn $\subseteq\left(N_{p} \times N_{r}\right) \cup\left(N_{r} \times N_{p}\right)$ indicates synapses between both proposition neurons and rule neurons. Two binary matrices $U=\left\{u_{i j}\right\}_{m \times n}$ and $V=\left\{v_{i j}\right\}_{m \times n}$, where $u_{i j}$ and $v_{i j} \in\{0,1\}$, are used to represent syn. $u_{i j}=1$ if there is a directed arc (synapse) from proposition neuron $\sigma_{p i}$ to rule neuron $\sigma_{r j}$; otherwise $u_{i j}=0 . v_{i j}=1$ if there is a directed arc (synapse) from rule neuron $\sigma_{r j}$ to proposition neuron $\sigma_{p i}$; otherwise $v_{i j}=0$. Note that there are no synapse connections between any two proposition neurons or between any two rule neurons; 
(5) $I$ and $O$ are input neuron set and output neuron set, respectively.

In the FRSN P systems, each proposition neuron corresponds to a proposition either in the antecedent part or consequent part of a fuzzy production rule, and each rule neuron corresponds to the category of antecedent part of a fuzzy production rule. Each neuron (proposition neuron or rule neuron) contains only one spiking (firing) rule, of the form $E / a^{x} \rightarrow a^{y}$, where $E=a^{z}$ is called the firing condition and $z$ is the number of input synapses from other neurons to the neuron. The firing condition $E=a^{z}$ indicates that if the neuron receives $z$ spikes the spiking rule can be applied; otherwise the rule cannot be enabled until $z$ spikes are received. For a neuron $\sigma_{i}$, if the firing rule $E / a^{x} \rightarrow a^{y}$ can be applied, it means that its pulse value $x>0$ is consumed (removed), and then it produces a spike with value $y$. Once the spike with value $y$ is excited from neuron $\sigma_{i}$, all neurons $\sigma_{j}$ with $(i, j) \subseteq$ syn will immediately receive the spike.

3.2. Matrix Based Reasoning Algorithm. The parallel reasoning process of FRSN P systems is implemented by a matrix execution algorithm. In order to describe the execution algorithm logically and concisely, the following operators and functions are used:

(1) $\oplus: C=A \oplus B$, where $A, B$, and $C$ are all $r \times s$ matrices; such that $c_{i j}=\max \left\{a_{i j}, b_{i j}\right\}$;

$(2) \otimes: C=A \otimes B$, where $A, B$, and $C$ are $r \times s$, $s \times t, r \times t$ matrices, respectively; such that $c_{i j}=$ $\max _{1 \leq k \leq s}\left\{a_{i k}, b_{k j}\right\}$;

(3) $\odot: C=A \odot B$, where $A, B$, and $C$ are $r \times s$, $s \times t, r \times t$ matrices, respectively; such that $c_{i j}=$ $\min _{1 \leq k \leq s}\left\{a_{i k}, b_{k j}\right\}$;

(4) $\beta=$ fire $(\alpha, \theta, \lambda)$, where $\beta=\left(\beta_{1}, \beta_{2}, \ldots, \beta_{r}\right)^{\mathrm{T}}, \alpha=$ $\left(\alpha_{1}, \alpha_{2}, \ldots, \alpha_{r}\right)^{\mathrm{T}}, \theta=\left(\theta_{1}, \theta_{2}, \ldots, \theta_{r}\right)^{\mathrm{T}}$, and $\lambda=$ $\left(\lambda_{1}, \lambda_{2}, \ldots, \lambda_{r}\right)^{\mathrm{T}}$. The function is defined as follows:

$$
\beta_{i}= \begin{cases}\alpha_{i}, & \text { if } \theta_{i}=\lambda_{i} \\ 0, & \text { if } \theta_{i}<\lambda_{i},\end{cases}
$$

where $i=1,2, \ldots, r$;

(5) $\beta=$ update $(\alpha, \theta, \lambda)$, where $\beta=\left(\beta_{1}, \beta_{2}, \ldots, \beta_{r}\right)^{\mathrm{T}}, \alpha=$ $\left(\alpha_{1}, \alpha_{2}, \ldots, \alpha_{r}\right)^{\mathrm{T}}, \theta=\left(\theta_{1}, \theta_{2}, \ldots, \theta_{r}\right)^{\mathrm{T}}, \lambda=$ $\left(\lambda_{1}, \lambda_{2}, \ldots, \lambda_{r}\right)^{\mathrm{T}}$. The function is defined as follows:

$$
\beta_{i}= \begin{cases}0, & \text { if } \theta_{i}=0 \\ \beta_{i}+\alpha_{i}, & \text { if } 0<\theta_{i}<\lambda_{i} \\ 0, & \text { if } \theta_{i}=\lambda_{i},\end{cases}
$$

where $i=1,2, \ldots, r$.

(6) $D=\operatorname{diag}(b)$, where $D=\left\{d_{i j}\right\}_{r \times r}$ is a $r \times r$ diagonal matrix and $b=\left(b_{1}, b_{2}, \ldots, b_{r}\right)$. For $1 \leq i \leq r, d_{i i}=b_{i}$, while $d_{i j}=0$ for $i \neq j$.
Then the matrix based reasoning algorithm for FRSN P systems can be described as follows [30].

Input. Parameter matrices $U=\left\{u_{i j}\right\}_{m \times n}, V=\left\{v_{i j}\right\}_{m \times n}, \Gamma=$ $\operatorname{diad}\left\{\gamma_{r 1}, \gamma_{r 2}, \ldots, \gamma_{r n}\right\}, H=\operatorname{diad}\left\{h_{r 1}, h_{r 2}, \ldots, h_{r n}\right\}, G=\operatorname{diad}$ $\left\{g_{r 1}, g_{r 2}, \ldots, g_{r n}\right\}, \lambda_{p}=\operatorname{diad}\left\{\lambda_{p 1}, \lambda_{p 2}, \ldots, \lambda_{p m}\right\}^{\mathrm{T}}, \lambda_{r}=$ $\operatorname{diad}\left\{\lambda_{r 1}, \lambda_{r 2}, \ldots, \lambda_{r n}\right\}^{\mathrm{T}}$, and initial inputs $\alpha_{p}^{0}=$ $\left(\alpha_{p 1}^{0}, \alpha_{p 2}^{0}, \ldots, \alpha_{p m}^{0}\right)^{\mathrm{T}}, \theta_{p}^{0}=\left(\theta_{p 1,}^{0}, \theta_{p 2}^{0}, \ldots, \theta_{p m}^{0}\right)^{\mathrm{T}}$, where $\operatorname{diad}\{\}$ stands for the diagonal matrix.

Output. The fuzzy truth values of propositions associated with the neurons in $O$.

Step 1. Let $\alpha_{r}^{0}=(0,0, \ldots, 0)^{\mathrm{T}}, \theta_{r}^{0}=(0,0, \ldots, 0)^{\mathrm{T}}$.

Step 2. Let $l=0$.

Step 3. (1) Process the firing of proposition neurons as follows:

$$
\begin{gathered}
\beta_{p}^{l}=\operatorname{fire}\left(\alpha_{p}^{l}, \theta_{p}^{l}, \lambda_{p}\right), \quad b_{p}^{l}=\operatorname{fire}\left(1, \theta_{p}^{l}, \lambda_{p}\right), \\
B_{p}^{l}=\operatorname{diag}\left(b_{p}^{l}\right), \\
\alpha_{p}^{l}=\operatorname{update}\left(\alpha_{p}^{l}, \theta_{p}^{l}, \lambda_{p}\right), \quad \theta_{p}^{l}=\operatorname{update}\left(\theta_{p}^{l}, \theta_{p}^{l}, \lambda_{p}\right) .
\end{gathered}
$$

(2) Compute the truth values of rule neurons and the number of received spikes.

$$
\begin{aligned}
& \alpha_{r}^{l+1}=\alpha_{r}^{l} \oplus\left[\left(H \times\left(\left(B_{p}^{l} \times U\right)^{\mathrm{T}} \odot \beta_{p}^{l}\right)\right)\right. \\
&\left.+\left(G \times\left(\left(B_{p}^{l} \times U\right)^{\mathrm{T}} \otimes \beta_{p}^{l}\right)\right)\right], \\
& \theta_{r}^{l+1}=\theta_{r}^{l}+\left[\left(B_{p}^{l} \times U\right)^{\mathrm{T}} \times b_{p}^{l}\right] .
\end{aligned}
$$

(3) Process the firing of rule neurons as follows:

$$
\begin{gathered}
\beta_{r}^{l+1}=\text { fire }\left(\Gamma \times \alpha_{r}^{l+1}, \theta_{r}^{l+1}, \lambda_{r}\right), \quad b_{r}^{l+1}=\text { fire }\left(1, \theta_{r}^{l+1}, \lambda_{r}\right), \\
B_{r}^{l+1}=\operatorname{diag}\left(b_{r}^{l+1}\right), \\
\alpha_{r}^{l+1}=\operatorname{update}\left(\alpha_{r}^{l+1}, \theta_{r}^{l+1}, \lambda_{r}\right), \\
\theta_{r}^{l+1}=\operatorname{update}\left(\theta_{r}^{l+1}, \theta_{r}^{l+1}, \lambda_{r}\right) .
\end{gathered}
$$

(4) Compute the truth values of proposition neurons and the number of received spikes as follows:

$$
\begin{aligned}
& \alpha_{p}^{l+1}=\alpha_{p}^{l} \oplus\left[\left(V \times B_{r}^{l+1}\right) \otimes \beta_{r}^{l+1}\right], \\
& \theta_{p}^{l+1}=\theta_{p}^{l}+\left[\left(V \times B_{r}^{l+1}\right) \times b_{r}^{l+1}\right] .
\end{aligned}
$$

Step 4. If $\theta_{p}^{l+1}=(0,0, \ldots, 0)^{\mathrm{T}}, \theta_{r}^{l+1}=(0,0, \ldots, 0)^{\mathrm{T}}$ (stopping criteria), reasoning results are obtained; otherwise, $l=l+1$, and go to Step 3. 


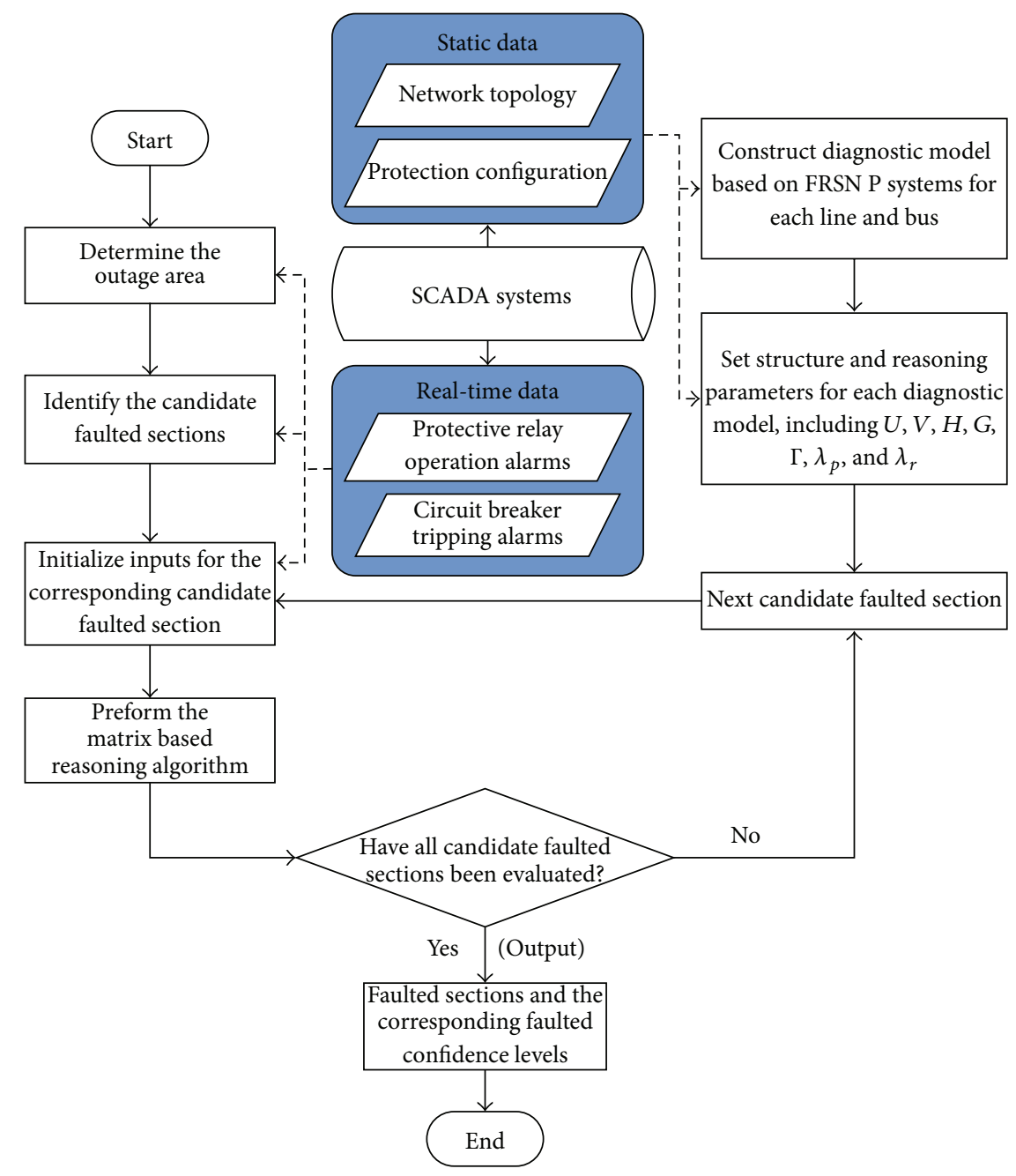

FIGURE 2: Developed diagnostic framework based on FRSN P systems.

\section{Implementing FRSN P Systems for Fault Diagnosis of Power Systems}

4.1. Diagnostic Framework. The developed diagnostic framework for fault diagnosis of power systems using FRSN P systems is shown in Figure 2. It can be seen that this framework mainly consists of three modules: one to identify the candidate faulted sections, one to construct the diagnostic models, and one to calculate the faulted confidence level. For the first module, because all the faulted sections undoubtedly locate in the outage area, the candidate faulted sections can therefore be identified via a real-time network topology determination method [32] which is based on both static data and real-time data derived from the SCADA systems. By using this method, the number of sections that needs to be diagnosed will decrease dramatically and thus reduce the subsequent computational burden. As for the third module, that is, calculation of the faulted confidence level of candidate faulted sections, the matrix based reasoning algorithm described in Section 3.2 can perform well. Another module, that is, the second module, is the most important module and is discussed in the next subsection. Note that the construction of this module only uses the static data, that is, the network topology and protection configuration. Consequently, this module can be constructed in advance and stored in files. And it can be easily modified according to the change of static data.

4.2. Diagnostic Model and Simulation Parameters Setting. A proper diagnostic model is prerequisite and critical to the sound diagnostic performance. It should be able to represent the causalities between faults and the actions of protective devices explicitly, which is beneficial to reasoning and understanding. Moreover, it can tackle the incompleteness and uncertainty of fault information well. In order to illustrate in detail, two FRSN P systems based diagnostic models associated with the fault scenarios of the line $\mathrm{L} 1$ and the bus $\mathrm{C}$ described in Section 2 are constructed and shown in Figure 3. Note that the developed diagnostic models are constructed adopting a backward reasoning strategy [15]. This strategy has three advantages. First, it can facilitate executing the matrix based reasoning algorithm of FRSN P systems and can instantly and directly obtain the corresponding faulted confidence level once the algorithm is finished. Second, the 


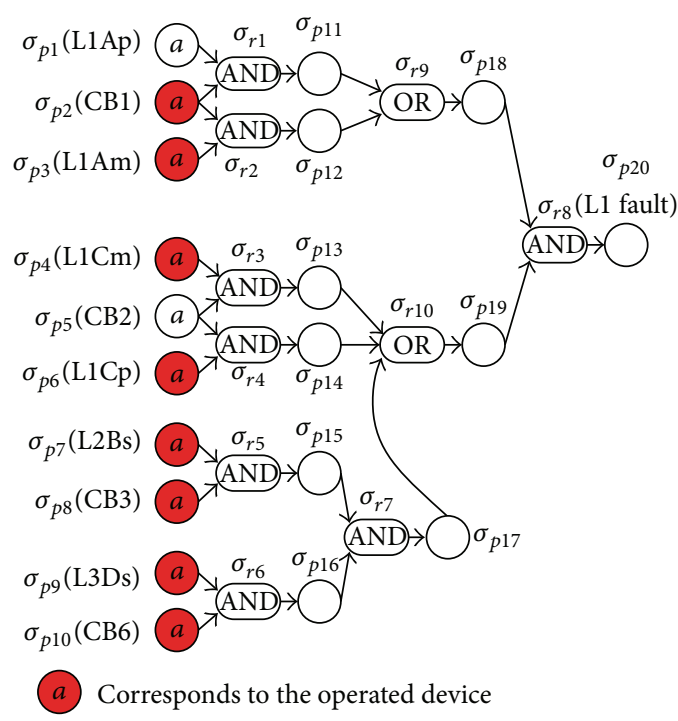

(a)

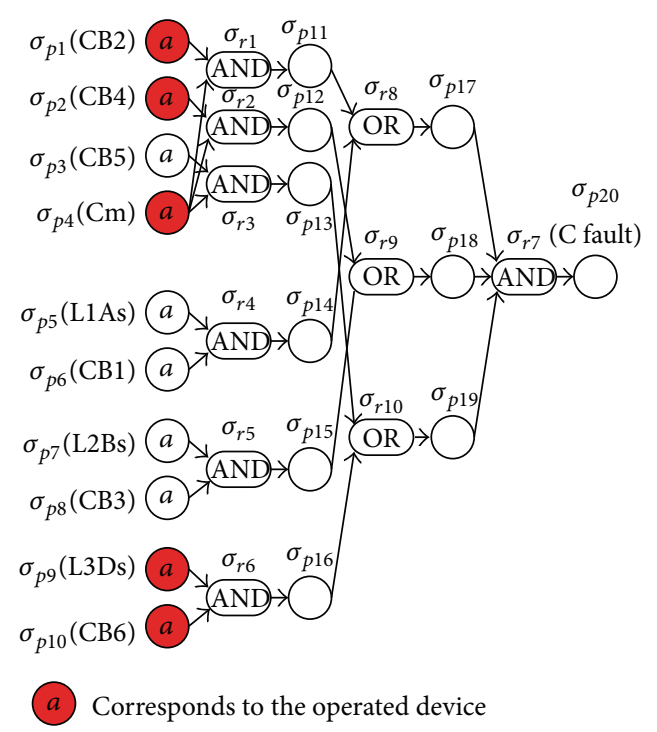

(b)

FIGURE 3: FRSN P systems based diagnostic models by means of SCADA data. (a) For the fault scenario of line L1; (b) for the fault scenario of bus C.

TABLE 1: Operation statistics of main protections of China state grid in $220 \mathrm{kV}$ and above power systems from 2004 to 2009.

\begin{tabular}{|c|c|c|c|c|c|c|c|}
\hline Year & 2004 & 2005 & 2006 & 2007 & 2008 & 2009 & Average \\
\hline \multicolumn{8}{|l|}{ Line } \\
\hline Total faults & 29411 & 29291 & 26047 & 24685 & 36865 & 12347 & 26441 \\
\hline Correct diagnosed faults & 29181 & 29234 & 26030 & 24666 & 36855 & 12345 & 26385.17 \\
\hline Diagnostic accuracy/\% & 99.22 & 99.81 & 99.93 & 99.92 & 99.97 & 99.98 & 99.79 \\
\hline \multicolumn{8}{|l|}{ Bus } \\
\hline Total faults & 90 & 120 & 112 & 122 & 159 & 89 & 115.33 \\
\hline Correct diagnosed faults & 77 & 114 & 102 & 118 & 156 & 88 & 109.17 \\
\hline Diagnostic accuracy/\% & 85.56 & 95.00 & 91.07 & 96.72 & 98.11 & 98.88 & 94.65 \\
\hline
\end{tabular}

diagnostic model is constructed for each section of a given power system, which reduces the scale of the model. Third, the "AND-OR" structure with the help of FRSN P systems is able to represent all possible combinations of main, primary backup, and secondary backup protection operations for reasoning a fault.

In Figure $3(\mathrm{a}), \sigma_{p 1}, \sigma_{p 2}, \ldots, \sigma_{p 20}$ are proposition neurons, where $\sigma_{p 1}, \sigma_{p 2}, \ldots, \sigma_{p 10}$ are input neurons and represent the protective devices associated with the line L1; $\sigma_{p 20}$ is an output neuron and represents the proposition "a fault occurs on the line L1." Considering the fault information has characteristics of incompleteness and uncertainty, fuzzy technology is employed to describe the truth degrees of the operation status of protective devices. If the operation of a protective device is actually observed from the SCADA system, the proposition will have a truth degree value $\alpha_{p i}^{0}=$ 0.90 ; otherwise, the proposition will have a truth degree value $\alpha_{p i}^{0}=0.10$.

The neurons $\sigma_{r 1}, \sigma_{r 2}, \ldots, \sigma_{r 10}$ in Figure $3(\mathrm{a})$ are rule neurons, where $\sigma_{r 1}, \sigma_{r 2}, \ldots, \sigma_{r 8}$ are "AND" type rule neurons and $\sigma_{r 9}, \sigma_{r 10}$ are "OR" type rule neurons. Each rule neuron is associated with a confidence factor $\gamma_{r i}$ to represent the confidence level of the production rule. In order to faithfully reflect the practical operation level of protective devices, the operation statistics $[33,34]$ of the state grid of China from year 2004 to year 2009 are used and shown in Table 1. All these statistics are the operation level of main protections and obtained from $220 \mathrm{kV}$ and above power systems. Since these statistics do not contain the operation information of backup protections, their operation levels are set via experience. In normal situation, the possibility of tripping main protections is higher than that of backup protections, and the possibility of tripping the primary backup protections is higher than that of the second backup protections [14]. In this context, the operation levels of primary backup and secondary backup are set as 0.85 and 0.80 , respectively. Therefore, the confidence factors $\gamma_{r 1}, \gamma_{r 2}, \gamma_{r 3}, \gamma_{r 4}, \gamma_{r 5}$, and $\gamma_{r 6}$ are given the values of $0.85,0.9979,0.9979,0.85,0.80,0.80$, respectively. The rest rule neurons $\gamma_{r 7}, \gamma_{r 8}, \gamma_{r 9}, \gamma_{r 10}$ are given the same value 1.0.

For the diagnostic model in Figure 3(b), the related parameters can be set by the same way. For example, the confidence factors $\gamma_{r 1}, \gamma_{r 2}, \gamma_{r 3}, \gamma_{r 4}, \gamma_{r 5}, \gamma_{r 6}, \gamma_{r 7}, \gamma_{r 8}, \gamma_{r 9}$, and $\gamma_{r 10}$ are given the values of $0.9465,0.9465,0.9465,0.80,0.80,0.80$, $1.0,1.0,1.0$, and 1.0 , respectively. 
4.3. Reasoning Process. After constructing the diagnostic model and setting the necessary simulation parameters, the matrix based reasoning algorithm can be used to pursue the faulted confidence level. Take the fault scenario of line L1 in Figure 1(a) as an example, according to the definition of FRSN $\mathrm{P}$ system, the relevant matrices and vectors associated with Figure 3(a) are as follows:

$$
\begin{aligned}
& U=\left[\begin{array}{llllllllllllllllllll}
1 & 1 & 0 & 0 & 0 & 0 & 0 & 0 & 0 & 0 & 0 & 0 & 0 & 0 & 0 & 0 & 0 & 0 & 0 & 0 \\
0 & 1 & 1 & 0 & 0 & 0 & 0 & 0 & 0 & 0 & 0 & 0 & 0 & 0 & 0 & 0 & 0 & 0 & 0 & 0 \\
0 & 0 & 0 & 1 & 1 & 0 & 0 & 0 & 0 & 0 & 0 & 0 & 0 & 0 & 0 & 0 & 0 & 0 & 0 & 0 \\
0 & 0 & 0 & 0 & 1 & 1 & 0 & 0 & 0 & 0 & 0 & 0 & 0 & 0 & 0 & 0 & 0 & 0 & 0 & 0 \\
0 & 0 & 0 & 0 & 0 & 0 & 1 & 1 & 0 & 0 & 0 & 0 & 0 & 0 & 0 & 0 & 0 & 0 & 0 & 0 \\
0 & 0 & 0 & 0 & 0 & 0 & 0 & 0 & 1 & 1 & 0 & 0 & 0 & 0 & 0 & 0 & 0 & 0 & 0 & 0 \\
0 & 0 & 0 & 0 & 0 & 0 & 0 & 0 & 0 & 0 & 0 & 0 & 0 & 0 & 1 & 1 & 0 & 0 & 0 & 0 \\
0 & 0 & 0 & 0 & 0 & 0 & 0 & 0 & 0 & 0 & 0 & 0 & 0 & 0 & 0 & 0 & 0 & 1 & 1 & 0 \\
0 & 0 & 0 & 0 & 0 & 0 & 0 & 0 & 0 & 0 & 1 & 1 & 0 & 0 & 0 & 0 & 0 & 0 & 0 & 0 \\
0 & 0 & 0 & 0 & 0 & 0 & 0 & 0 & 0 & 0 & 0 & 0 & 1 & 1 & 0 & 0 & 1 & 0 & 0 & 0
\end{array}\right]^{\mathrm{T}} \\
& V= \\
& \left.\begin{array}{llllllllllllllllllll}
0 & 0 & 0 & 0 & 0 & 0 & 0 & 0 & 0 & 0 & 1 & 0 & 0 & 0 & 0 & 0 & 0 & 0 & 0 & 0 \\
0 & 0 & 0 & 0 & 0 & 0 & 0 & 0 & 0 & 0 & 0 & 1 & 0 & 0 & 0 & 0 & 0 & 0 & 0 & 0 \\
0 & 0 & 0 & 0 & 0 & 0 & 0 & 0 & 0 & 0 & 0 & 0 & 1 & 0 & 0 & 0 & 0 & 0 & 0 & 0 \\
0 & 0 & 0 & 0 & 0 & 0 & 0 & 0 & 0 & 0 & 0 & 0 & 0 & 1 & 0 & 0 & 0 & 0 & 0 & 0 \\
0 & 0 & 0 & 0 & 0 & 0 & 0 & 0 & 0 & 0 & 0 & 0 & 0 & 0 & 1 & 0 & 0 & 0 & 0 & 0 \\
0 & 0 & 0 & 0 & 0 & 0 & 0 & 0 & 0 & 0 & 0 & 0 & 0 & 0 & 0 & 1 & 0 & 0 & 0 & 0 \\
0 & 0 & 0 & 0 & 0 & 0 & 0 & 0 & 0 & 0 & 0 & 0 & 0 & 0 & 0 & 0 & 1 & 0 & 0 & 0 \\
0 & 0 & 0 & 0 & 0 & 0 & 0 & 0 & 0 & 0 & 0 & 0 & 0 & 0 & 0 & 0 & 0 & 0 & 0 & 1 \\
0 & 0 & 0 & 0 & 0 & 0 & 0 & 0 & 0 & 0 & 0 & 0 & 0 & 0 & 0 & 0 & 0 & 1 & 0 & 0 \\
0 & 0 & 0 & 0 & 0 & 0 & 0 & 0 & 0 & 0 & 0 & 0 & 0 & 0 & 0 & 0 & 0 & 0 & 1 & 0
\end{array}\right]^{\mathrm{T}},
\end{aligned}
$$$$
H=\left[\begin{array}{llllllllll}
1 & 0 & 0 & 0 & 0 & 0 & 0 & 0 & 0 & 0 \\
0 & 1 & 0 & 0 & 0 & 0 & 0 & 0 & 0 & 0 \\
0 & 0 & 1 & 0 & 0 & 0 & 0 & 0 & 0 & 0 \\
0 & 0 & 0 & 1 & 0 & 0 & 0 & 0 & 0 & 0 \\
0 & 0 & 0 & 0 & 1 & 0 & 0 & 0 & 0 & 0 \\
0 & 0 & 0 & 0 & 0 & 1 & 0 & 0 & 0 & 0 \\
0 & 0 & 0 & 0 & 0 & 0 & 1 & 0 & 0 & 0 \\
0 & 0 & 0 & 0 & 0 & 0 & 0 & 1 & 0 & 0 \\
0 & 0 & 0 & 0 & 0 & 0 & 0 & 0 & 0 & 0 \\
0 & 0 & 0 & 0 & 0 & 0 & 0 & 0 & 0 & 0
\end{array}\right],
$$$$
G=\left[\begin{array}{llllllllll}
0 & 0 & 0 & 0 & 0 & 0 & 0 & 0 & 0 & 0 \\
0 & 0 & 0 & 0 & 0 & 0 & 0 & 0 & 0 & 0 \\
0 & 0 & 0 & 0 & 0 & 0 & 0 & 0 & 0 & 0 \\
0 & 0 & 0 & 0 & 0 & 0 & 0 & 0 & 0 & 0 \\
0 & 0 & 0 & 0 & 0 & 0 & 0 & 0 & 0 & 0 \\
0 & 0 & 0 & 0 & 0 & 0 & 0 & 0 & 0 & 0 \\
0 & 0 & 0 & 0 & 0 & 0 & 0 & 0 & 0 & 0 \\
0 & 0 & 0 & 0 & 0 & 0 & 0 & 0 & 0 & 0 \\
0 & 0 & 0 & 0 & 0 & 0 & 0 & 0 & 1 & 0 \\
0 & 0 & 0 & 0 & 0 & 0 & 0 & 0 & 0 & 1
\end{array}\right],
$$$$
\Gamma=\left[\begin{array}{cccccccccc}
0.85 & 0 & 0 & 0 & 0 & 0 & 0 & 0 & 0 & 0 \\
0 & 0.9979 & 0 & 0 & 0 & 0 & 0 & 0 & 0 & 0 \\
0 & 0 & 0.9979 & 0 & 0 & 0 & 0 & 0 & 0 & 0 \\
0 & 0 & 0 & 0.85 & 0 & 0 & 0 & 0 & 0 & 0 \\
0 & 0 & 0 & 0 & 0.80 & 0 & 0 & 0 & 0 & 0 \\
0 & 0 & 0 & 0 & 0 & 0.80 & 0 & 0 & 0 & 0 \\
0 & 0 & 0 & 0 & 0 & 0 & 1.0 & 0 & 0 & 0 \\
0 & 0 & 0 & 0 & 0 & 0 & 0 & 1.0 & 0 & 0 \\
0 & 0 & 0 & 0 & 0 & 0 & 0 & 0 & 1.0 & 0 \\
0 & 0 & 0 & 0 & 0 & 0 & 0 & 0 & 0 & 1.0
\end{array}\right]
$$$$
\lambda_{p}=\left(\begin{array}{llllllllllllllllllll}
1 & 1 & 1 & 1 & 1 & 1 & 1 & 1 & 1 & 1 & 1 & 1 & 1 & 1 & 1 & 1 & 1 & 1 & 1 & 1
\end{array}\right)^{\mathrm{T}},
$$

$$
\lambda_{r}=\left(\begin{array}{llllllllll}
2 & 2 & 2 & 2 & 2 & 2 & 2 & 2 & 2 & 3
\end{array}\right)^{\mathrm{T}} .
$$

The initial inputs are

$\alpha_{p}^{0}=\quad \begin{array}{llllllllll}0.10 & 0.90 & 0.90 & 0.90 & 0.10 & 0.90 & 0.90 & 0.90 & 0.90 & 0.90\end{array}$

$$
\begin{array}{llllllllll}
0 & 0 & 0 & 0 & 0 & 0 & 0 & 0 & 0 & 0)^{\mathrm{T}},
\end{array}
$$

$\theta_{p}^{0}=\left(\begin{array}{llllllllllllllllllll}1 & 1 & 1 & 1 & 1 & 1 & 1 & 1 & 1 & 1 & 0 & 0 & 0 & 0 & 0 & 0 & 0 & 0 & 0 & 0\end{array}\right)^{\mathrm{T}}$.

Let

$$
\begin{aligned}
& \alpha_{r}^{0}=\left(\begin{array}{llllllllll}
0 & 0 & 0 & 0 & 0 & 0 & 0 & 0 & 0 & 0
\end{array}\right)^{\mathrm{T}}, \\
& \theta_{r}^{0}=\left(\begin{array}{llllllllll}
0 & 0 & 0 & 0 & 0 & 0 & 0 & 0 & 0 & 0
\end{array}\right)^{\mathrm{T}} .
\end{aligned}
$$

According to the matrix based reasoning algorithm described previously, the reasoning steps will result in the following.

(1) The first reasoning step

$$
\begin{aligned}
& \alpha_{p}^{1}=\left(\begin{array}{lllllllllll}
0 & 0 & 0 & 0 & 0 & 0 & 0 & 0 & 0 & 0 & 0
\end{array}\right. \\
& \begin{array}{llllllllll}
0.0850 & 0.8981 & 0.0998 & 0.0850 & 0.720 & 0.720 & 0 & 0 & 0 & 0)^{\mathrm{T}},
\end{array}, \\
& \theta_{p}^{1}=\left(\begin{array}{llllllllllllllllllll}
0 & 0 & 0 & 0 & 0 & 0 & 0 & 0 & 0 & 0 & 1 & 1 & 1 & 1 & 1 & 1 & 0 & 0 & 0 & 0
\end{array}\right)^{\mathrm{T}}, \\
& \alpha_{r}^{1}=\left(\begin{array}{llllllllll}
0 & 0 & 0 & 0 & 0 & 0 & 0 & 0 & 0 & 0
\end{array}\right)^{\mathrm{T}}, \\
& \theta_{r}^{1}=\left(\begin{array}{llllllllll}
0 & 0 & 0 & 0 & 0 & 0 & 0 & 0 & 0 & 0
\end{array}\right)^{\mathrm{T}} \text {. }
\end{aligned}
$$

(2) The second reasoning step

$$
\begin{gathered}
\alpha_{p}^{2}=\left(\begin{array}{cccccccccccccccccccc}
0 & 0 & 0 & 0 & 0 & 0 & 0 & 0 & 0 & 0 & 0 & 0 & 0 & 0 & 0 & 0 & 0.720 & 0.8981 & 0 & 0
\end{array}\right)^{\mathrm{T}}, \\
\theta_{p}^{2}=\left(\begin{array}{llllllllllllllllllll}
0 & 0 & 0 & 0 & 0 & 0 & 0 & 0 & 0 & 0 & 0 & 0 & 0 & 0 & 0 & 0 & 1 & 1 & 0 & 0
\end{array}\right)^{\mathrm{T}} \\
\alpha_{r}^{2}= \\
\theta_{r}^{2}=\left(\begin{array}{lllllllllll}
0 & 0 & 0 & 0 & 0 & 0 & 0 & 0 & 0 & 0.0998
\end{array}\right)^{\mathrm{T}} \\
0
\end{gathered}
$$

(3) The third reasoning step

$$
\begin{gathered}
\alpha_{p}^{3}=\left(\begin{array}{llllllllllllllllllll}
0 & 0 & 0 & 0 & 0 & 0 & 0 & 0 & 0 & 0 & 0 & 0 & 0 & 0 & 0 & 0 & 0 & 0 & 0.720 & 0
\end{array}\right)^{\mathrm{T}}, \\
\theta_{p}^{3}=\left(\begin{array}{llllllllllllllllllll}
0 & 0 & 0 & 0 & 0 & 0 & 0 & 0 & 0 & 0 & 0 & 0 & 0 & 0 & 0 & 0 & 0 & 0 & 1 & 0
\end{array}\right)^{\mathrm{T}} \\
\alpha_{r}^{3}= \\
\theta_{r}^{3}=\left(\begin{array}{lllllllllllll}
0 & 0 & 0 & 0 & 0 & 0 & 0 & 0.8981 & 0 & 0
\end{array}\right)^{\mathrm{T}} \\
0
\end{gathered}
$$

(4) The fourth reasoning step

$$
\begin{aligned}
& \alpha_{p}^{4}=\left(\begin{array}{llllllllllllllllllll}
0 & 0 & 0 & 0 & 0 & 0 & 0 & 0 & 0 & 0 & 0 & 0 & 0 & 0 & 0 & 0 & 0 & 0 & 0 & 0.720
\end{array}\right)^{\mathrm{T}}, \\
& \theta_{p}^{4}=\left(\begin{array}{llllllllllllllllllll}
0 & 0 & 0 & 0 & 0 & 0 & 0 & 0 & 0 & 0 & 0 & 0 & 0 & 0 & 0 & 0 & 0 & 0 & 0 & 1
\end{array}\right)^{\mathrm{T}} \\
& \alpha_{r}^{4}=\left(\begin{array}{llllllllll}
0 & 0 & 0 & 0 & 0 & 0 & 0 & 0 & 0 & 0
\end{array}\right)^{\mathrm{T}} \\
& \theta_{r}^{4}=\left(\begin{array}{llllllllll}
0 & 0 & 0 & 0 & 0 & 0 & 0 & 0 & 0 & 0
\end{array}\right)^{\mathrm{T}} .
\end{aligned}
$$




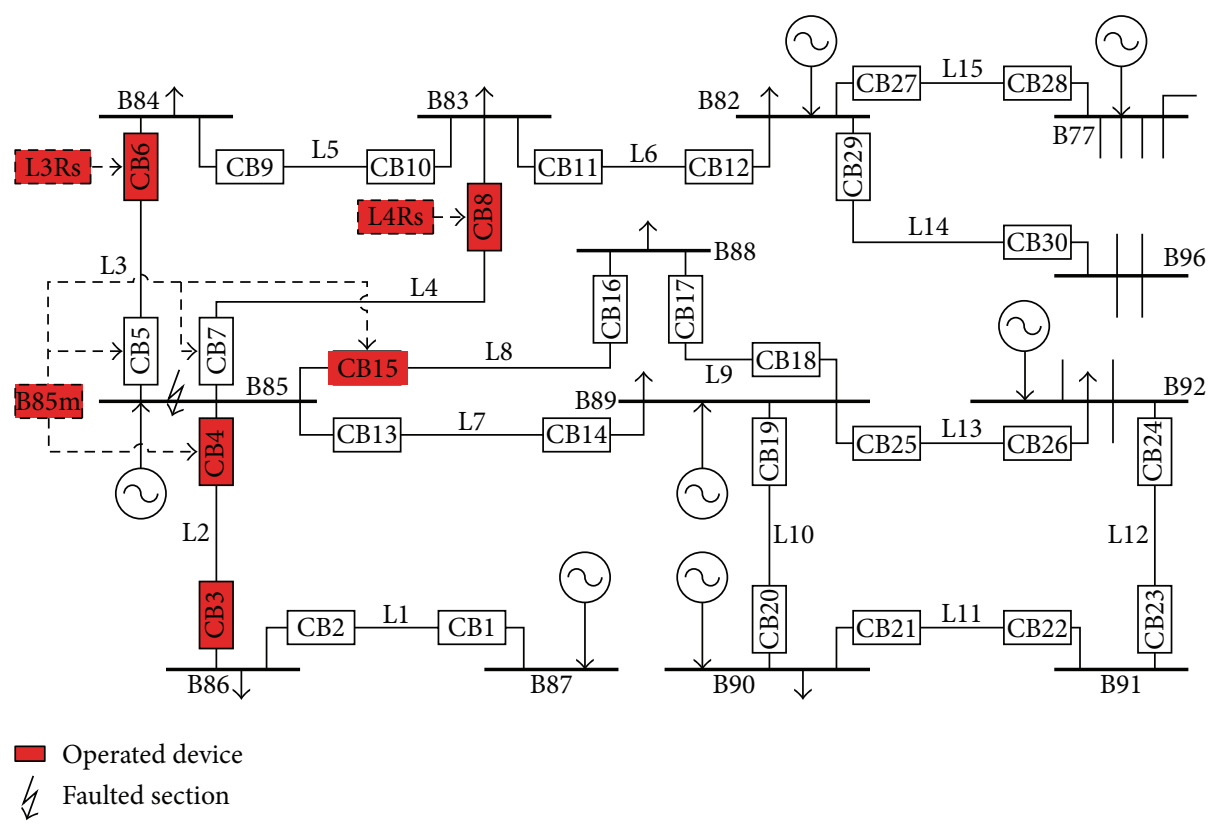

FIGURE 4: Subnetwork of IEEE 118-bus power system. R and S denote the power receiving and sending ends of a line, respectively.

(5) The fifth reasoning step

$$
\begin{gathered}
\theta_{p}^{5}=\left(\begin{array}{cccccccccccccccccccc}
0 & 0 & 0 & 0 & 0 & 0 & 0 & 0 & 0 & 0 & 0 & 0 & 0 & 0 & 0 & 0 & 0 & 0 & 0 & 0
\end{array}\right)^{\mathrm{T}}, \\
\theta_{r}^{5}=\left(\begin{array}{llllllllll}
0 & 0 & 0 & 0 & 0 & 0 & 0 & 0 & 0 & 0
\end{array}\right)^{\mathrm{T}} .
\end{gathered}
$$

Thus, since the system reaches the stopping criteria, it exports its reasoning result; that is, the truth value of the proposition $\sigma_{p 20}$ is 0.720 . A candidate faulted section with a faulted confidence level greater than 0.5 is regarded as a faulted section. So the conclusion is that a fault occurs on the line L1 with a confidence level 0.720 .

For the fault scenario of bus $\mathrm{C}$ in Figure 1(b) and its diagnostic model in Figure 3(b), the same construction and reasoning way can be used to pursue the diagnostic conclusion. The conclusion is that a fault occurs on the bus $\mathrm{C}$ with a confidence level 0.720 .

\section{Applications and Discussion}

In this section, three different power systems are employed to illustrate and validate the effectiveness of the proposed fault diagnosis approach.

\subsection{Applications and Results}

5.1.1. Application 1. The first test system, which is adopted from [35] and shown in Figure 4, is a subnetwork from the IEEE 118-bus system. It is employed to test single fault, involving failed protective devices and incorrect tripping signal. This system consists of 28 sections (13 buses: B77, B82 B92, B96, and 15 lines: L1 L15), 103 protective relays, and 30 circuit breakers. In this scenario, line L7 is in maintenance
TABLE 2: Observed SCADA data for application 1.

\begin{tabular}{lc}
\hline Sequence number & Observed signal \\
\hline 1 & Main protective relay B85m \\
2 & Secondary backup protective relay L3Rs \\
3 & Secondary backup protective relay L4Rs \\
4 & Circuit breaker CB3 \\
5 & Circuit breaker CB4 \\
6 & Circuit breaker CB6 \\
7 & Circuit breaker CB8 \\
8 & Circuit breaker CB15 \\
\hline
\end{tabular}

TABLE 3: Candidate faulted sections and diagnostic results for application 1 .

\begin{tabular}{lcc}
\hline Candidate faulted sections & $\begin{array}{c}\text { Faulted } \\
\text { confidence level }\end{array}$ & Faulted section \\
\hline Bus B85 & 0.720 & Yes \\
Line L2 & 0.0998 & No \\
Line L3 & 0.0998 & No \\
Line L4 & 0.0998 & No \\
\hline
\end{tabular}

and out of service. During the operation, a fault occurs, and the observed fault data from the SCADA system are listed in Table 2. According to the observed fault data, the candidate faulted sections can be identified and listed in Table 3. After identifying the candidate faulted sections, their FRSN P systems based diagnostic models can be quickly recalled from the storage files. A representative diagnostic model associated with bus B85 is shown in Figure 5. According to Figure 5, the relevant structure matrices and vectors associated with the diagnostic models can be obtained. With all preparations 


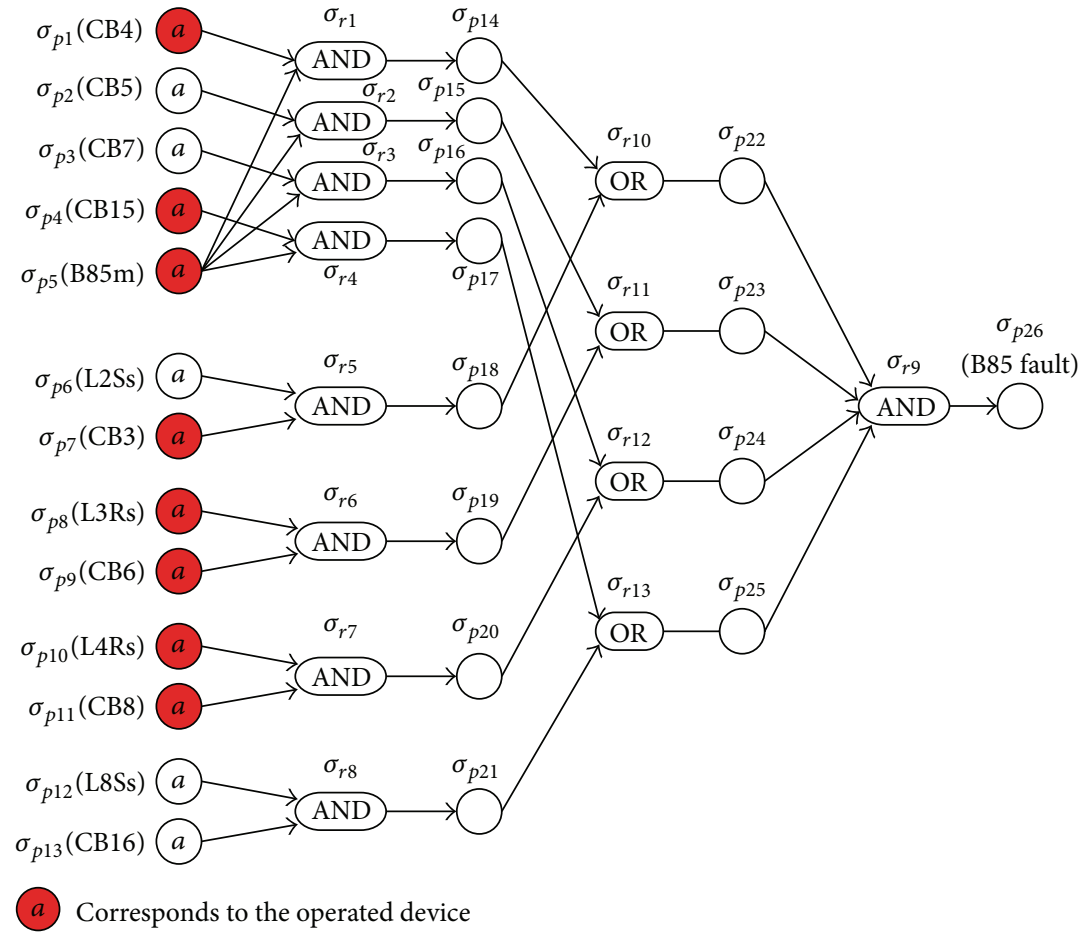

FIGURE 5: FRSN P systems based diagnostic model for application 1.

completed, the matrix based reasoning algorithm is executed on the diagnostic models and able to immediately achieve the diagnostic results. The diagnostic results are summarized in Table 3. Consequently, bus B85 is a faulted section with confidence level 0.720 . The fault scenario is detailed as follows: a fault occurs on bus B85 and the main protective relay $\mathrm{B} 85 \mathrm{~m}$ operates to send a signal to trip circuit breakers CB4, CB5, CB7, and CB15. CB4 and CB5 succeed in opening, while CB5 and CB7 fail. The corresponding secondary backup protective relays L3Rs and L4Rs then operate to successfully trip their associated circuit breakers CB6 and CB8 to clear the fault. For the observed signal CB3, it is an incorrect tripping signal which may be caused by communication error or malfunction.

5.1.2. Application 2. This fault scenario is adopted from [19]. The related power system and observed data from the SCADA system are shown in Figure 6 and Table 4, respectively. The fault scenario is mainly used to verify the capability in handling multiple faults with failed and malfunctioned devices. With the help of the observed data, the candidate faulted sections can be immediately identified and listed in Table 5. A representative FRSN P systems based diagnostic model is shown in Figure 7 . The diagnostic results are summarized in Table 5. It can be seen from the results that two faults occur on sections L29 and L30. The detailed fault clearance process is that a fault on section L30 causes L30's own main protective relays L30-23m and L30-24m to operate, thus successfully tripping circuit breakers CB60 and CB59, respectively. Meanwhile, another fault occurring on section
TABLE 4: Observed SCADA data for application 2.

\begin{tabular}{lc}
\hline Sequence number & Observed signal \\
\hline 1 & Main protective relay L29-23m \\
2 & Main protective relay L29-27m \\
3 & Main protective relay L30-23m \\
4 & Main protective relay L30-24m \\
5 & Primary backup protective relay L29-23p \\
6 & Primary backup protective relay L29-27p \\
7 & Secondary backup protective relay L25-20s \\
8 & Circuit breaker CB50 \\
9 & Circuit breaker CB57 \\
10 & Circuit breaker CB58 \\
11 & Circuit breaker CB59 \\
12 & Circuit breaker CB60 \\
\hline
\end{tabular}

TABle 5: Candidate faulted sections and diagnostic results for application 2 .

\begin{tabular}{lcc}
\hline Candidate faulted sections & $\begin{array}{c}\text { Faulted } \\
\text { confidence level }\end{array}$ & Faulted section \\
\hline Bus BUS23 & 0.0947 & No \\
Bus BUS24 & 0.0947 & No \\
Bus BUS27 & 0.0947 & No \\
Line L25 & 0.0998 & No \\
Line L29 & 0.8981 & Yes \\
Line L30 & 0.8981 & Yes \\
\hline
\end{tabular}




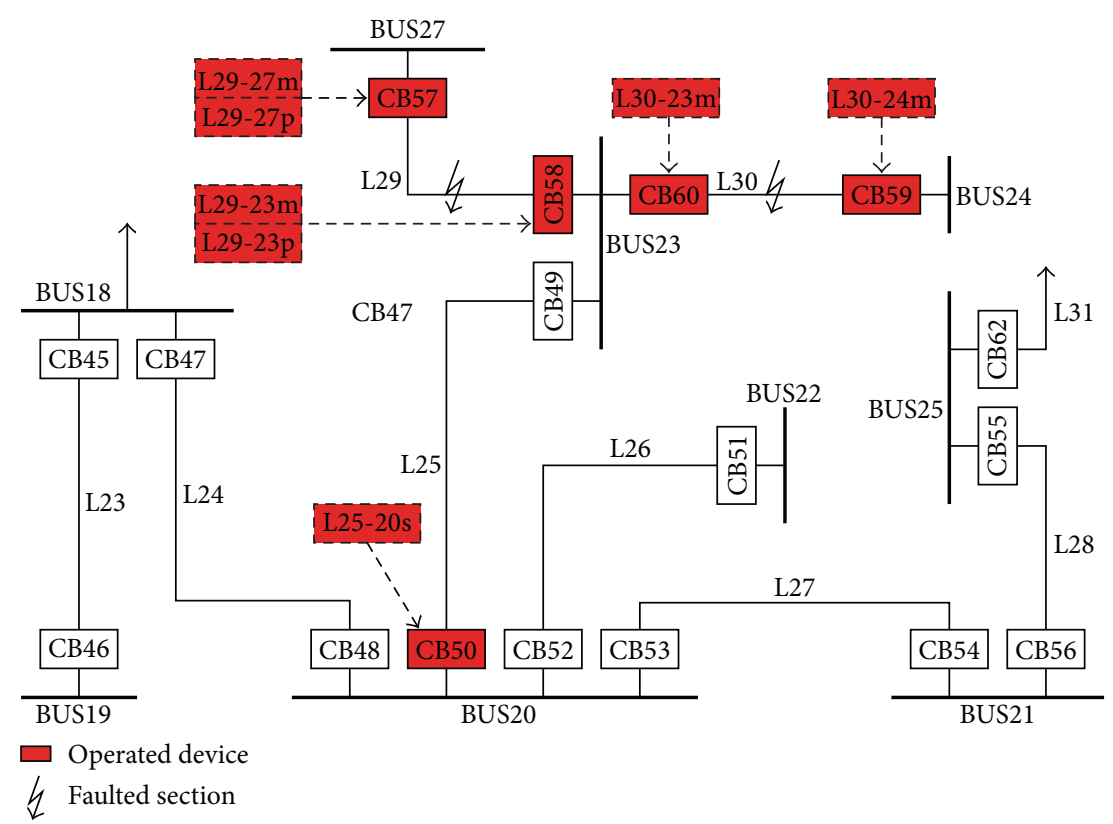

Figure 6: Nine-bus power system.

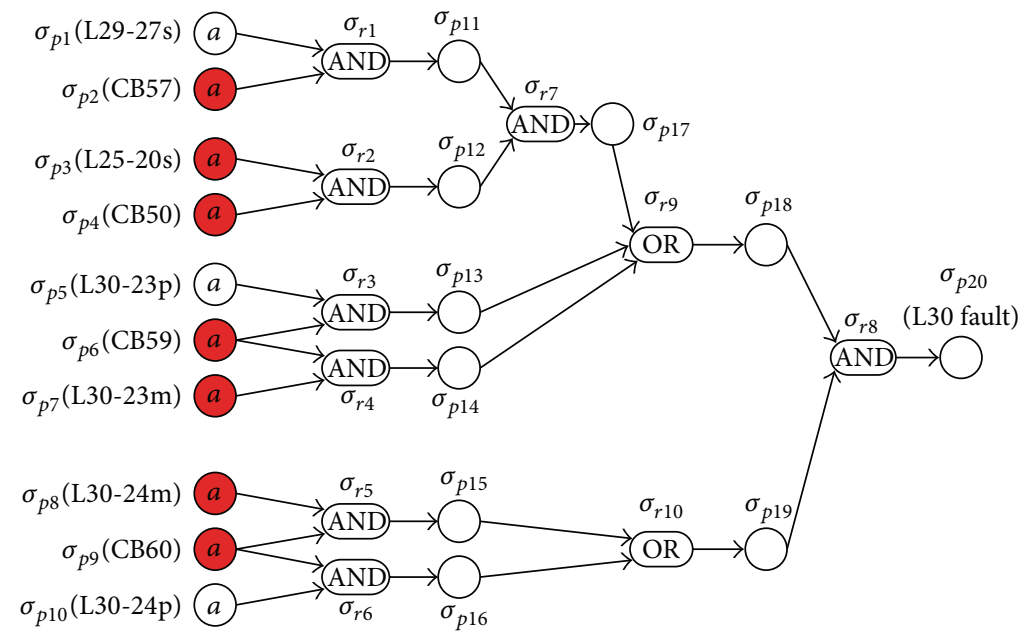

Corresponds to the operated device

FIGURE 7: FRSN P systems based diagnostic model for application 2.

L29 leads to the operations of main protective relays L29$23 \mathrm{~m}$ and L29-27m. However, the operations fail to open the circuit breakers CB57 and CB58. It is because of this failure that the corresponding primary protective relays L29-23p and L29-27p operate to successfully trip CB57 and CB58 and then clear the fault. With regard to the signal L25-20s, it is a malfunctioned data which results in the tripping of circuit breaker CB50.

5.1.3. Application 3. To further confirm the validity of the proposed fault diagnosis approach, another power system in Figure 8 is tested. This system, adopted from [15], is a 14-bus system consisting of 14 buses (B01 B14) and 20 lines (L0102 L1413). The protection configuration consists of 134 protective relays and 40 circuit breakers. Numerous fault scenarios containing single and multiple faults involving failed and/or malfunctioned protective devices are investigated. Table 6 lists some typical scenarios with the corresponding diagnostic results. Among these scenarios, the first four are single fault cases. In scenario 1, all protective relays and circuit breakers operate correctly. The following two scenarios are designed based on scenario 1 . Scenario 2 is designed to investigate the failure of protective device (CB1314), and scenario 3 is designed to investigate the malfunction of protective device (L1413m). Scenario 4 is designed to simultaneously investigate the failure (L1213m, L1312m) and malfunction (L0613s) of protective devices. From Table 6, it can be seen that the proposed fault diagnosis approach is able to diagnose single 


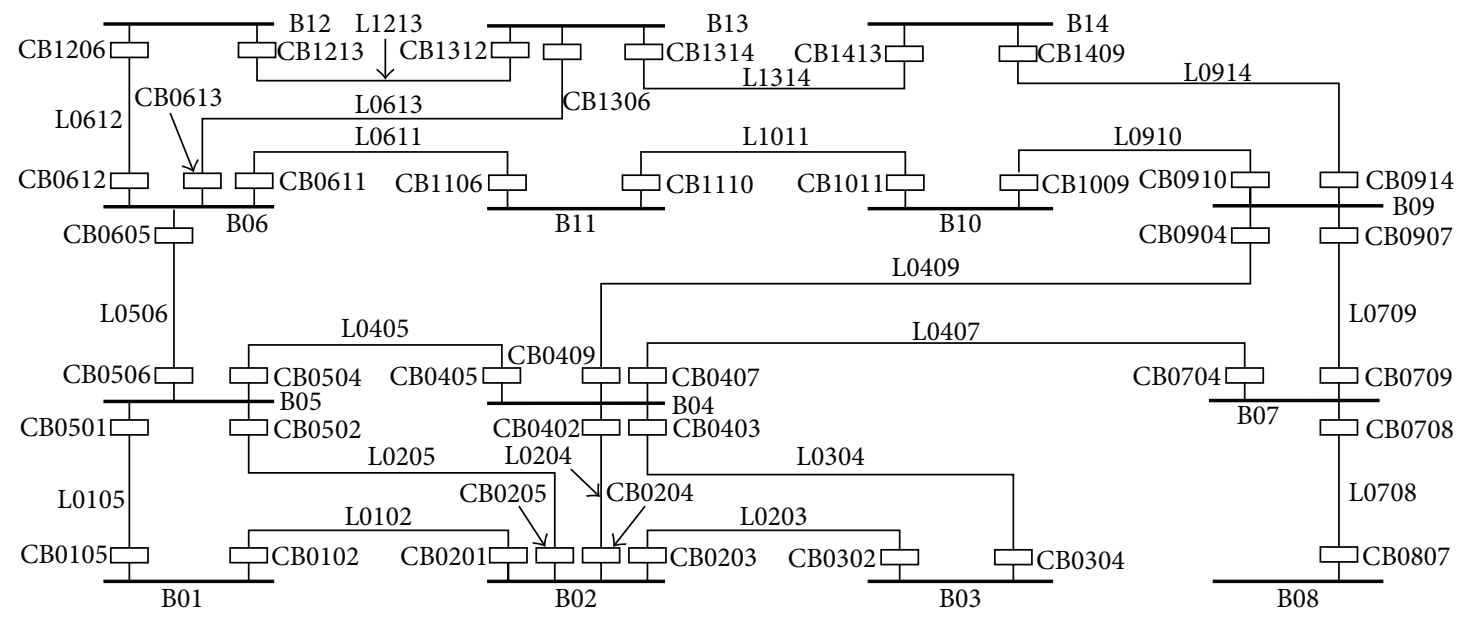

Figure 8: 14-bus power system.

TABLE 6: Typical fault scenarios and the corresponding diagnostic results for application 3.

\begin{tabular}{llc}
\hline Scenario & Observed signal & Faulted section/confidence level \\
\hline 1 & B13m, CB1306, CB1312, CB1314 & B13/0.8519 \\
2 & B13m, L1413s, CB1306, CB1312, CB1413 & B13/0.720 \\
3 & B13m, L1413m, CB1306, CB1312, CB1314 & B13/0.8519 \\
4 & L1213p, L1312p, L0613s, CB0613, CB1213, CB1312 & $\mathrm{L} 1213 / 0.765$ \\
5 & B04m, B09m, CB0402, CB0405, CB0407, CB0409, & B04/0.8519, B09/0.8519 \\
6 & CB0904, CB0907, CB0910, CB0914 & B14/0.720, L1314/0.8981 \\
7 & B14m, L1314m, L1413m, L0914s, CB0914, CB1314, CB1413 & B10/0.720, B11/0.8519 \\
\end{tabular}

fault scenarios with failure and/or malfunction of protective devices. The last three scenarios are multiple faults cases. In scenario 5, all protective devices operate correctly, and no false data is present. In scenario 6, a circuit breaker (CB1409) fails to open, and the fault is cleared by the corresponding secondary backup protective devices. The next fault scenario is more complex. It is coupled with many protective devices failing to work properly. Although multiple faults case puts forward higher demand on fault tolerant capability of fault diagnosis approach, it can be seen from Table 6 that the proposed approach can still enjoy excellent diagnostic effect.

5.2. Discussion. FRSN P systems are a novel visual model for representing fuzzy reasoning rules. In this work, they are employed to diagnose the faulted section(s) of power systems. It can be summarized from the diagnostic results that

(1) since the FRSN P systems based diagnostic model is constructed for each section of a given power system, its scale is independent of the scale of the power system but dependent on the connection schemes between the section and its adjacent lines;

(2) the matrix based reasoning algorithm of FRSN P systems is able to obtain the diagnostic results by at most five reasoning steps (five steps for line, four steps for bus). Moreover, the number of reasoning steps is not connected with the breadth of the FRSN P systems based diagnostic model, but with the depth;

(3) compared with the main existing fault diagnosis approaches, the proposed approach presents the benefits:

(a) Compared with ES, it needs no heavy procedures of knowledge acquisition and knowledge base maintenance. Because the scale of the developed FRSN P systems based diagnostic model is constructed for each section of a given power system, so it has better flexibility versus the change of the power system's topology. Additionally, it is able to handle uncertainty problems and thus has better fault tolerant capability, and its response time is very short.

(b) Compared with ANNs, it does not require the construction of a comprehensive training data and the execution of a complicated training process, so it has neither a problem of convergence nor a tedious determination of network's dimension and weights. Besides, it can explicitly represent the causalities between the sections and the actions of protective devices and thus help dispatchers to understand and analyze the 
fault evolution and clearance processes. Furthermore, it has better flexibility versus the change of the power system's topology.

(c) Compared with BNs, it does not require the construction of a comprehensive training data and the execution of a complicated training process; thus it has no problem of convergence.

(d) Compared with PNs, on the surface, PNs and FRSN P systems are similar. Both PNs and FRSN $\mathrm{P}$ systems have the characteristics of graphic discrete event representation and parallel information reasoning. However, the FRSN P systems possess more different types of versatile neurons, including proposition neuron, "AND" type rule neuron and "OR" type rule neuron. While Petri nets only contain place and transition. In this context, FRSN P systems have better flexibility and extensibility. Besides, these versatile neurons of the FRSN P systems are able to represent the "AND-OR" causalities among the PRs, CBs, and power system sections more explicitly and visually for dispatchers. Thus, it is doubtlessly in favor of dispatchers' comprehension, analysis, and summary on the fault evolution and clearance processes. In addition, these two models have other essential differences which have been discussed in detail in [36].

(e) Compared with CE-Nets, the backward reasoning strategy enables the FRSN P systems based diagnostic model to visually represent all possible combinations of main, primary backup and secondary backup protection operations for reasoning a fault. In addition, it has better fault tolerant capability.

(f) Compared with AOM, it does not need to design an elaborate mathematical model for modeling the operation logic of protective devices. Additionally, the solution methods for AOM are also avoided; thus there is no concern regarding the convergence problem.

\section{Conclusion and Future Work}

This paper has proposed a novel approach based on FRSN $\mathrm{P}$ systems for fault diagnosis of power systems. FRSN P systems possess the characteristics of graphic discrete event representation, dynamic firing mechanism, and parallel reasoning. They are capable of representing information containing uncertainty and performing fuzzy reasoning through matrix operation. Three different applications are used to verify the feasibility and effectiveness of the proposed fault diagnosis approach. The diagnostic results demonstrate that this approach is able to diagnose different single and multiple faults coupled with failed and/or malfunctioned protective devices. Since it requires only simple matrix operation and has good fault tolerance, the proposed approach is very suitable for integration and management with the existing
SCADA systems and is used as a reliable tool for fault diagnosis of power systems. Additionally, because the developed FRSN P systems based diagnostic model can be constructed in advance, and stored in files, and can obtain the diagnostic results by no more than five reasoning steps, it is especially suitable for online application. In future, how to realize the online application and how the FRSN P systems based diagnostic model adapts to the change of power system's topology are our further research work.

\section{Acknowledgments}

The authors would like to thank the editors and the reviewers for their constructive comments. This work was supported by the National Natural Science Foundation of China (no. 51107048).

\section{References}

[1] T. Minakawa, Y. Ichikawa, M. Kunugi, K. Shimada, N. Wada, and M. Utsunomiya, "Development and implementation of a power system fault diagnosis expert system," IEEE Transactions on Power Systems, vol. 10, no. 2, pp. 932-940, 1995.

[2] Y. M. Park, G. W. Kim, and J. M. Sohn, "A logic based expert system (LBES) for fault diagnosis of power system," IEEE Transactions on Power Systems, vol. 12, no. 1, pp. 363-369, 1997.

[3] H. J. Lee, B. S. Ahn, and Y. M. Park, "A fault diagnosis expert system for distribution substations," IEEE Transactions on Power Delivery, vol. 15, no. 1, pp. 92-97, 2000.

[4] T. Bi, Z. Yan, F. Wen et al., "On-line fault section estimation in power systems with radial basis function neural network," International Journal of Electrical Power and Energy Systems, vol. 24, no. 4, pp. 321-328, 2002.

[5] T. Bi, F. Wen, Y. Ni, and F. F. Wu, "Distributed fault section estimation system using radial basis function neural network and its companion fuzzy system," International Journal of Electrical Power and Energy System, vol. 25, no. 5, pp. 377-386, 2003.

[6] W. M. Lin, C. H. Lin, and Z. C. Sun, "Event-orthogonal errorinsensitive multiple fault detection with cascade correlation network," IEEE Transactions on Power Delivery, vol. 18, no. 4, pp. 1152-1157, 2003.

[7] J. C. S. de Souza, E. M. Meza, M. T. Schilling, and M. B. D. C. Filho, "Alarm processing in electrical power systems through a neuro-fuzzy approach," IEEE Transactions on Power Delivery, vol. 19, no. 2, pp. 537-544, 2004.

[8] G. Cardoso, J. G. Rolim, and H. H. Zurn, "Identifying the primary fault section after contingencies in bulk power systems," IEEE Transactions on Power Delivery, vol. 23, no. 3, pp. 13351342,2008

[9] Z. He, J. Zhao, J. Yang, and W. Gao, "A new power system fault diagnosis method based on rough set theory and quantum neural network," in Proceedings of the Asia-Pacific Power and Energy Engineering Conference (APPEEC '09), pp. 1-4, Wuhan, China, March 2009.

[10] Y. Zhu, L. Huo, and J. Lu, "Bayesian networks-based approach for power systems fault diagnosis," IEEE Transactions on Power Delivery, vol. 21, no. 2, pp. 634-639, 2006.

[11] L. He, "Application of Bayesian network in power grid fault diagnosis," in Proceedings of the 4th International Conference on Natural Computation (ICNC '08), pp. 61-64, Jinan, China, October 2008. 
[12] M. M. Mansour, M. A. A. Wahab, and W. M. Soliman, "Bayesian networks for fault diagnosis of a large power station and its transmission lines," Electric Power Components and Systems, vol. 40, no. 8, pp. 845-863, 2012.

[13] C. N. Hadjicostis and G. C. Verghese, "Power system monitoring using Petri net embeddings," IEE Proceedings: Generation, Transmission and Distribution, vol. 147, no. 5, pp. 299-303, 2000.

[14] J. Sun, S. Qin, and Y. Song, "Fault diagnosis of electric power systems based on Fuzzy Petri Nets," IEEE Transactions on Power Systems, vol. 19, no. 4, pp. 2053-2059, 2004.

[15] X. Luo and M. Kezunovic, "Implementing fuzzy reasoning Petri-nets for fault section estimation," IEEE Transactions on Power Delivery, vol. 23, no. 2, pp. 676-685, 2008.

[16] J. Yang, Z. He, X. Tan, and Q. Zeng, "A distributed fault diagnosis approach in power system based on fuzzy reasoning petri net," in Proceedings of the Asia-Pacific Power and Energy Engineering Conference (APPEEC '10), pp. 1-4, Chengdu, China, March 2010.

[17] A. T. Ghainani, A. A. M. Zin, and N. A. M. Ismail, "Fuzzy timing Petri net for fault diagnosis in power system," Mathematical Problems in Engineering, vol. 2012, Article ID 717195, 12 pages, 2012.

[18] W. H. Chen, C. W. Liu, and M. S. Tsai, "On-line fault diagnosis of distribution substations using hybrid cause-effect network and fuzzy rule-based method," IEEE Transactions on Power Delivery, vol. 15, no. 2, pp. 710-717, 2000.

[19] W. H. Chen, S. H. Tsai, and H. I. Lin, "Fault section estimation for power networks using logic cause-effect models," IEEE Transactions on Power Delivery, vol. 26, no. 2, pp. 963-971, 2011.

[20] F. Wen and C. S. Chang, "A tabu search approach to fault section estimation in power systems," Electric Power Systems Research, vol. 40, no. 1, pp. 63-73, 1997.

[21] Y. Liu, Y. Li, Y. Cao, and C. Guo, "Forward and backward models for fault diagnosis based on parallel genetic algorithms," Journal of Zhejiang University: Science A, vol. 9, no. 10, pp. 1420-1425, 2008.

[22] Z. He, H. D. Chiang, C. Li, and Q. Zeng, "Fault-section estimation in power systems based on improved optimization model and binary particle swarm optimization," in Proceedings of the IEEE Power and Energy Society General Meeting (PES '09), pp. 1-8, Calgary, Canada, July 2009.

[23] X. Lin, S. Ke, Z. Li, H. Weng, and X. Han, "A fault diagnosis method of power systems based on improved objective function and genetic algorithm-tabu search," IEEE Transactions on Power Delivery, vol. 25, no. 3, pp. 1268-1274, 2010.

[24] W. Guo, F. Wen, G. Ledwich, Z. Liao, X. He, and J. Liang, "An analytic model for fault diagnosis in power systems considering malfunctions of protective relays and circuit breakers," IEEE Transactions on Power Delivery, vol. 25, no. 3, pp. 1393-1401, 2010.

[25] S. J. Huang and X. Z. Liu, "Application of artificial bee colony-based optimization for fault section estimation in power systems," International Journal of Electrical Power and Energy Systems, vol. 44, no. 1, pp. 210-218, 2013.

[26] M. Ionescu, G. Pǎun, and T. Yokomori, "Spiking neural $\mathrm{P}$ systems," Fundamenta Informaticae, vol. 71, no. 2-3, pp. 279308, 2006.

[27] G. Păun, "Computing with membranes," Journal of Computer and System Sciences, vol. 61, no. 1, pp. 108-143, 2000.

[28] T. Wang, J. Wang, H. Peng, and H. Wang, "Knowledge representation and reasoning based on FRSN P system," in Proceedings of the 9th World Congress on Intelligent Control and Automation (WCICA '11), pp. 849-854, Taipei City, Taiwan, 2011.
[29] J. Wang, L. Zou, H. Peng, and G. Zhang, "An extended spiking neural P system for fuzzy knowledge representation," International Journal of Innovative Computing, Information and Control, vol. 7, no. 7, pp. 3709-3724, 2011.

[30] H. Peng, J. Wang, M. J. Pérez-Jiménez, H. Wang, J. Shao, and T. Wang, "Fuzzy reasoning spiking neural $\mathrm{P}$ system for fault diagnosis," vol. 235, pp. 106-116.

[31] H. J. Cho and J. K. Park, "An expert system for fault section diagnosis of power systems using fuzzy relations," IEEE Transactions on Power Systems, vol. 12, no. 1, pp. 342-348, 1997.

[32] M. Prais and A. Bose, "Topology processor that tracks network modifications over time," IEEE Transactions on Power Systems, vol. 3, no. 3, pp. 992-998, 1988.

[33] X. Shen, Z. Shu, Y. Liu, P. Lü, and L. Zhang, "Statistics and analysis on operation situation of protective relayings of state grid corporation of China in 2009," Power System Technology, vol. 35, no. 2, pp. 189-193, 2011.

[34] X. Shen, Z. Shu, Y. Liu, P. Lü, and L. Zhang, "Statistics and analysis on operation situation of protective relayings of state grid corporation of China in 2008," Power System Technology, vol. 34, no. 3, pp. 173-177, 2010.

[35] H. Ren and Z. Mi, "Power system fault diagnosis modeling techniques based on encoded petri nets," in Proceedings of the IEEE Power Engineering Society General Meeting (PES '06), pp. 1111-1118, Montreal, Canada, June 2006.

[36] J. Wang, P. Shi, H. Peng, M. Perez-Jimenez, and T. Wang, "Weighted fuzzy spiking neural P systems," IEEE Transactions on Fuzzy Systems, vol. 21, no. 2, pp. 209-220, 2013. 


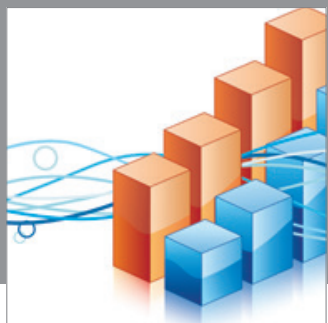

Advances in

Operations Research

mansans

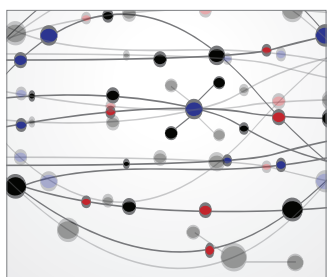

The Scientific World Journal
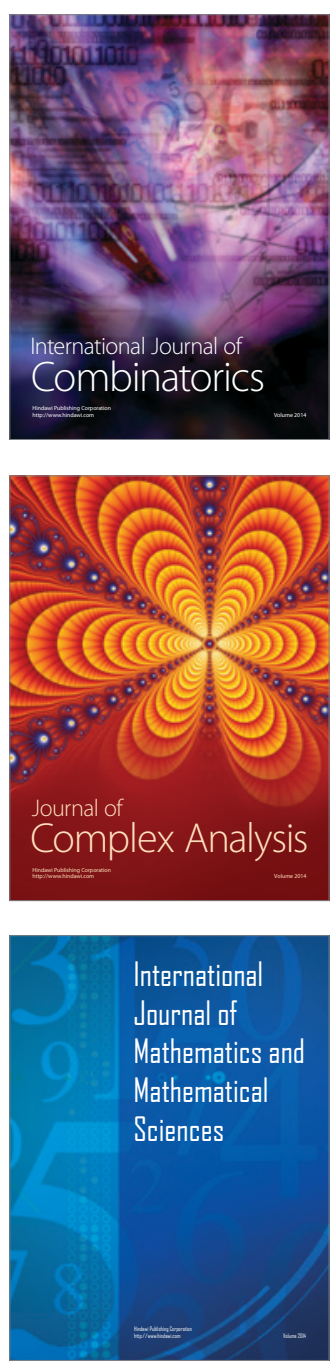
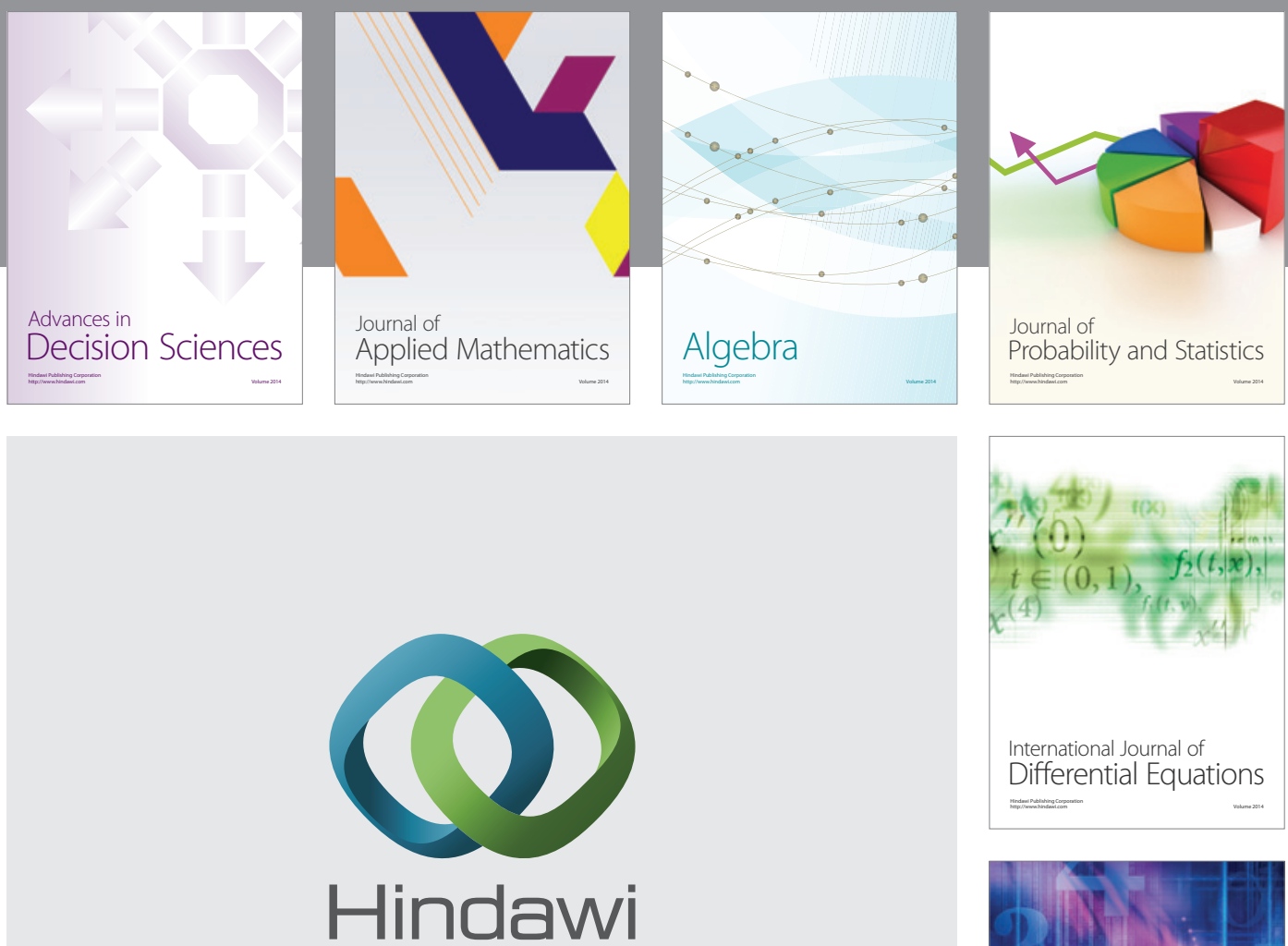

Submit your manuscripts at http://www.hindawi.com
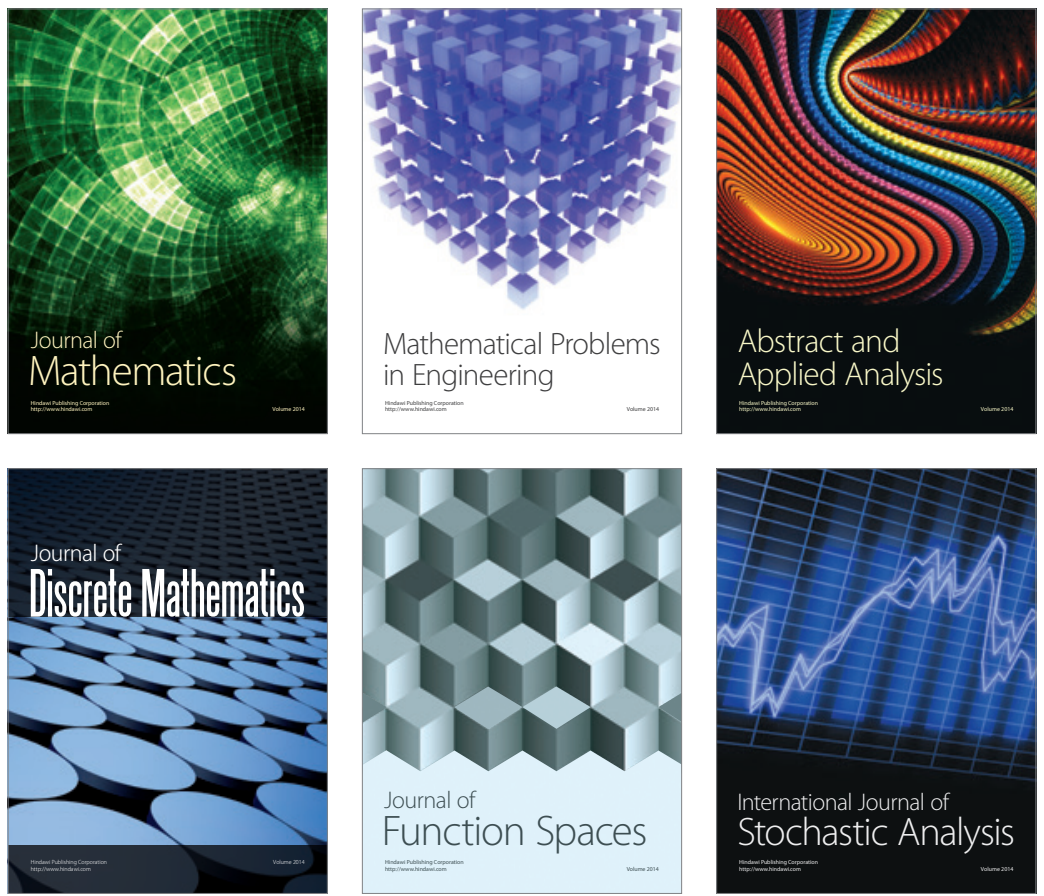

Journal of

Function Spaces

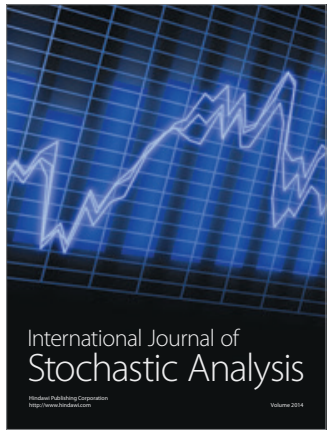

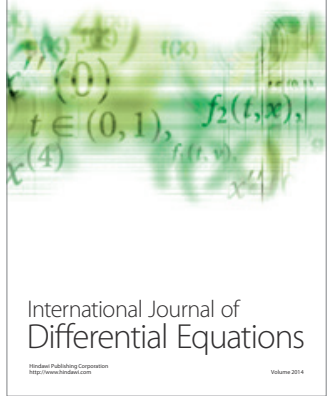
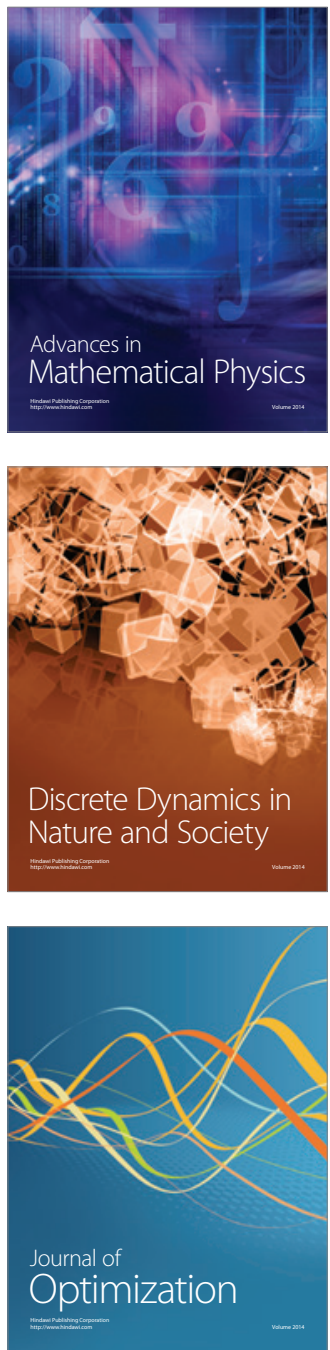